\title{
Study on Test Generation Method for Analog Circuit Board Based on Rough Set
}

\author{
Chenggang Wang, Xinghai Wang and Jiaqi Chen \\ School of Basic Science for Aviation, Naval Aviation University, Yantai 264001, China
}

\begin{abstract}
In order to improve the repair and maintenance efficiency for analog circuit boards of certain equipment, a test and diagnosis system was designed based on virtual instrument. System structure is introduced briefly. As the key technique of the system, stimuli generation is studied emphatically. Fault simulation method based on SPICE is introduced firstly. Then test analysis and generation method based on rough set is interpreted on the basis of fault simulation. By decision table matrix reduction, test points are optimized, and fault diagnosis strategy is designed. Moreover, the AND-THEN rules generated by rough set are used in circuit fault detection and isolation. Application proves that the system has high efficiency and precision.
\end{abstract}

Keywords-virtual instrument; fault simulation; diagnosis strategy; rough set

\section{INTRODUCTION}

Circuit board is the basic unit of navy warship equipment. The maintenance of circuit board plays an important role in navy equipment technique support. As circuit boards have become increasingly dependent on complex electronic systems, the necessity of testing them to ensure reliable performance has increased in a similar fashion. It is estimated that testing presently accounts for $30-50 \%$ of an electronic manufacturer's cost for a product. This percentage, in fact, actually fails to show the true cost of testing for many systems, because it ignores the cost of maintenance efforts. The repair and test for circuit boards of certain equipment are accomplished by technicians with help of normal instrument. In this course, there are many defects such as time asynchronous, effect of human factors, low efficiency, etc. Because of various test items, technician must have adequate experience. A test and diagnosis system is designed based on virtual instrument.

As the key technique of the system, stimuli generation is studied emphatically. Fault simulation method based on SPICE is introduced firstly. Then test analysis and generation method based on rough set is interpreted on the basis of fault simulation. By decision table matrix reduction, test points are optimized, and fault diagnosis strategy can be designed. Moreover, the AND-THEN rules generated by rough set can be used in circuit fault detection and isolation.

Compared to traditional test method, our test and diagnosis system can fulfill the test course automatically with shorter development cycle and lower cost of research. Application proves that the system has high precision, good operability, and high intelligence. With the system, we can ensure the exertion of battle effectiveness adequately.

\section{SYSTEM COMPOSITION AND FAULT SIMULATION}

\section{A. Test and Diagnosis System Composition}

This system takes LabWindows/CVI as software development platform. Compared to other software platforms, the integrated development environment, interactive programming method, function panel and abundant function library enhance its function greatly [1]. Especially, with the aid of its signal processing capability in advanced analysis library, we can analyze and process acquired data expediently.

Our circuit board test and diagnosis system is made up of multifunction data acquisition (DAQ) device, GPIB devices (signal generator and oscillograph) and master computer. It realizes test and diagnosis for circuit boards by fault diagnosis system. The composition diagram of the system is shown in Fig1.

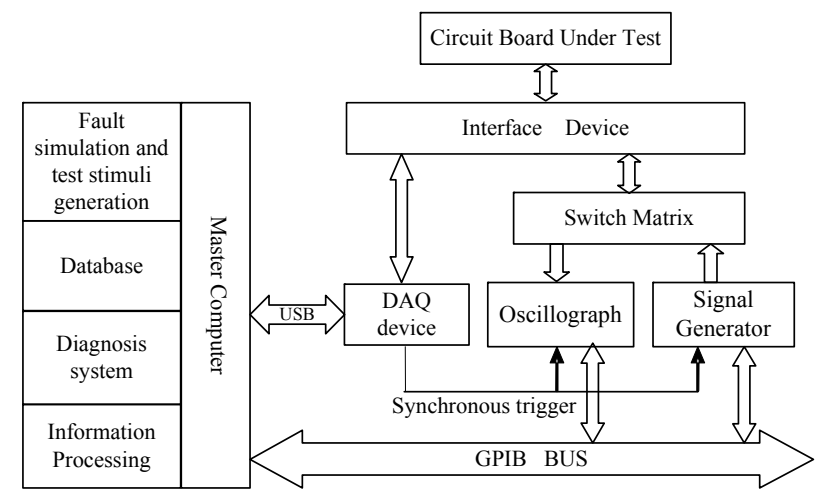

FIGURE I. SYSTEM COMPOSITION DIAGRAM

\section{B. Fault Simulation and Test Stimuli Generation}

A fault modeling method of physical fault of basic element and functional fault of integrated circuit is proposed and a technology based on PSPICE for fault modeling of simulation of analog circuit is discussed in [1][2]. In [3][4][5], The general process of analog circuit fault knowledge acquisition by fault simulation was summarized. We get the analog stimuli for diagnosis system by fault simulation. The test generation diagram is shown in Fig.2. 


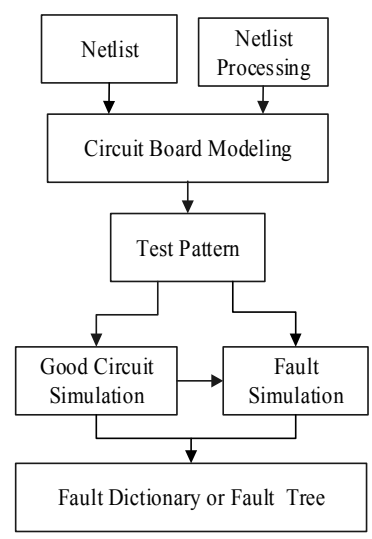

FIGURE II. TEST GENERATION FOR ANALOG CIRCUIT BOARD

Our fault simulator is developed on the basis of SPICE 3 simulation engine. The method of auto-acquiring knowledge was described in brief. The scheme of universal analog circuit fault knowledge acquisition flat was proposed. This flat could auto-enumerate all possible faults of circuit, simulate them by using external circuit simulator, extract and simplify the fault knowledge from the experiment result, and then form the structural fault knowledge basement.

Each component is defined by a set of nominal device and model parameter attributes, as well as parametric tolerances. Each component also has a set of associated failure modes. We edit the predefined failure modes or add our own catastrophic or parametric failure modes. Failure modes simulated programmatically generate the proper SPICE 3 syntax to describe the failure. Failure modes can be setup for primitive (resistor, transistor, etc.) as well as subcircuit elements. Any node on a part can be shorted to any other node, opened, or stuck [6][7]. Partial failure modes are shown in Table I. Other failure modes modeling methods are similar. For subcircuit elements, we define their failure modes in subcircuit model library.

TABLE I. SPICE3 SYNTAX FOR SOME FAILURE MODES

\begin{tabular}{|c|c|c|}
\hline Fault & Before Fault Injection & After Fault Injection \\
\hline Open Resistor & R9 $4530 \mathrm{~K}$ & R9 45100 MEG \\
\hline Short Resistor & R9 $4530 \mathrm{~K}$ & R9 45.1 \\
\hline Open Capacitor & C1 $111210 \mathrm{U}$ & R_C1 1112 100MEG \\
\hline Short Capacitor & C1 $111210 \mathrm{U}$ & R_C1 1112.1 \\
\hline Open Inductance & L1 $202110 \mathrm{M}$ & R_L1 2021100 MEG \\
\hline Short Inductance & L1 $202110 \mathrm{M}$ & R_L1 2021.1 \\
\hline Shorted Base Emitter & Q1 $1219242 \mathrm{~N} 1893$ & $\begin{array}{l}\text { Q1 } 121924 \text { 2N1893 } \\
\text { Rshort_19 } 1924.1\end{array}$ \\
\hline Low Beta Parametric fault & $\begin{array}{l}\text { Q1 } 121924 \text { QN2222 } \\
. \text { MODEL QN2222 NPN AF=1 BF=105 BR=4 } \\
\text { CJC=15.2P CJE=29.5P... }\end{array}$ & $\begin{array}{l}\text { Q1 } 1219 \text { 24 Q1_Fail } \\
. \text { MODEL Q1_Fail NPN AF=1 BF=10 BR=4 } \\
\text { CJC=15.2P CJE }=29.5 P \ldots\end{array}$ \\
\hline Resistor Stuck 2V below Vcc & R1 $601 \mathrm{~K}$ & $\begin{array}{l}\text { R1 } 601 \mathrm{~K} \\
\text { Rstuck_66_Stuck } 610.00000 \\
\text { Bstuck_6 6_Stuck 0 V=Vcc- } 2\end{array}$ \\
\hline
\end{tabular}

Fault simulation includes three steps: fault modeling, fault collapsing and fault injection. We select faults and inject them into circuit model with fault modeling and fault collapsing techniques. Then, analog stimulus is applied to the circuit and its response is recorded.

The simulation results for each failure are input into integrated database. They constitute the fault dictionary or fault tree, which are used in our test and diagnosis system.

\section{Fault Simulation Data Analysis}

Fault simulation data of each test point obtained as a vector. Its main features are:

(1) The characteristics and dimensions of each Vector are different. They cannot be normalized to the space to handle the hyper sphere.

(2) The number of concentrated vector is variable.

(3) There may be more vectors that can be classified or repetitive, but the class center is randomized. There may be sparser in the spatial distribution, self-contained class vector.
(4) Prior knowledge about the shape of class may be obtained, but the prior knowledge of class distribution cannot be obtained.

(5) The data of a dimension may be very close, even identical;

(6) The number of class classified may be big, but less data available.

We can see that the classification used must have a pattern search capabilities, greater flexibility, for outliers and noise insensitive. Taking above problems into account, this paper applies rough set to emulation information classification, analysis and evaluation test.

\section{TEST GENERATION BASED ON Rough SET}

Rough set theory was put forward by Polish mathematician Z. Pawlak in 1982 [8]. It use known knowledge to characterize the imprecise or uncertain knowledge (approximately). The most significant difference between rough set and other theories in dealing with the problem of uncertainty and imprecision is 
that it does not require any prior information. So of the problem or the procession of uncertainty can be described more objectively.

\section{A. Rough Set Theory}

An information system is the basic vehicle for data representation in the rough set framework. It is a single flat table in this context, either physically or logically in form of a view across several underlying tables. We can thus define an information system in terms of a pair $\langle\mathrm{U}, \mathrm{A}\rangle$. $\mathrm{U}$ is a non-empty finite set of objects and $\mathrm{A}$ is a non-empty finite set of attributes.

$$
S=<U, A, V, f>
$$

The corresponding information generated by the domain of information systems is the test signal for the $\mathrm{C}$ of condition attributes. Test generation is the process of rough set attribute reduction and classification problems essentially.

\section{B. Discernibility Matrix and Discernibility}

In (1), $|U|=n$, the discernibility matrix of $\mathrm{S}$ is $n \times n$. A transparent method to find the core and reduces of an information system $\mathrm{S}$ via discernibility matrix was given in [9]:

$$
\alpha(x, y)=\{a \in A \mid f(x, a) \neq f(y, a)\}
$$

For each attribute $a \in A$, we denote a Boolean variable "a". If $\alpha(x, y)=\left\{a_{1}, a_{2}, \mathrm{~L}, a_{k}\right\} \neq \phi$, then denote a Boolean function $a_{1} \vee a_{2} \vee \mathrm{L} \vee a_{k}$, which is illustrated by $\sum \alpha(x, y)$

If $\alpha(x, y)=\phi$, then a Boolean constant is denoted to 1 . Define discernibility by

$$
\Delta=\prod_{(x, y) \in U \times U} \sum \alpha(x, y)
$$

\section{Information Discretization}

Fault simulation results are processed discretely, with the symbols of each discrete conditional attribute (i.e., test signal) of the segment values[10][11]. The natural number $i\left(1 \leq i \leq n_{i}\right)$ represents the test signal segments after reduction. Then decision table matrix is obtained and expressed by matrix $K^{\prime}$. The rows of $K^{\prime}$ are the measured values of the test points with a fault mode (the number of representatives of the natural sub-test signal); the columns are measurements of a test signal with all failure mode.

Discretization of continuous attributes is the process of breakpoint on the attributes with the process of space division.
There are equidistant partition method, such as equal frequency partition, Navie Scaler, SemiNavie Scaler, logical reasoning and other heuristic algorithms.

In this paper, a heuristic algorithm for constrained discrete constrained is put forward. It can be summarized as three steps:

(1) The minimum resolution of test signal distance is taken as constraints, which is used to calculate candidate cut set.

(2) The importance of candidate cuts is measured through some heuristic method, and selects a subset as small as possible according to measurement results from the candidate cut set, which is used as practical discrete cut set.

(3) Cut set is applied in decision table and the actual information system is discretized.

\section{EXPERIMENTAL RESULTS AND ANALYSIS}

A pulse peak demodulation circuit is illustrated in Fig.3[12]. Port 2 and port 6 of U1, port 6 of U2, port 2, port 3 and port 6 of U3 are selected as test points. As shown in table II, 38 hard faults of resistor, capacitor, diode, triode and op amp, 17 typical soft faults are simulated. In figure 1, PULSE is pulse signal (test stimulus) with cycles $1 \mathrm{~ms}$, pulse width $4 \mu \mathrm{s}$, peak $0.5 \mathrm{~V}$. OFF is pulse signal with cycle $1 \mathrm{~ms}$, pulse width $90 \mu \mathrm{s}$, peak $5 \mathrm{~V}$.

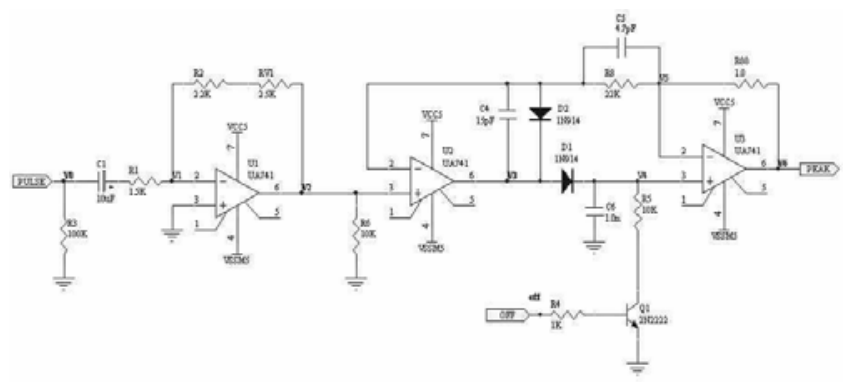

\begin{tabular}{|c|c|c|}
\hline Fault & Fault list & Number \\
\hline open & $\begin{array}{l}\mathrm{R} 1 / \mathrm{R} 2 / \mathrm{R} 3 / \mathrm{R} 4 / \mathrm{R} 5 / \mathrm{R} 6 / \mathrm{R} 7 / \mathrm{R} 8 / \mathrm{R} 88 / \mathrm{RV} \\
\text { 1/C1/C4/C5/C6/D1/D2/Q1_C/Q1_B/ } \\
\mathrm{Q} 1 \_E\end{array}$ & 19 \\
\hline short & $\begin{array}{l}\mathrm{R} 1 / \mathrm{R} 2 / \mathrm{R} 3 / \mathrm{R} 4 / \mathrm{R} 5 / \mathrm{R} 6 / \mathrm{R} 7 / \mathrm{R} 8 / \mathrm{R} 88 / \mathrm{RV} \\
\text { 1/C1/C4/C5/C6/D1/D2/Q1_BE/Q1_C } \\
\text { E/Q1_BC }\end{array}$ & 19 \\
\hline $\begin{array}{l}\text { Para } \\
\text { meter } \\
\text { Drifts }\end{array}$ & $\begin{array}{l}\mathrm{R} 1 / \mathrm{R} 2 / \mathrm{R} 3 / \mathrm{R} 4 / \mathrm{R} 5 / \mathrm{R} 6 / \mathrm{R} 7 / \mathrm{R} 8 / \mathrm{R} 88 / \mathrm{C} 1 / \\
\mathrm{C} 4 / \mathrm{C} 5 / \mathrm{C} 6 / \mathrm{U} 1 / \mathrm{U} 2 / \mathrm{U} 3 / \mathrm{Q} 1\end{array}$ & 17 \\
\hline
\end{tabular}

FIGURE III. A PULSE PEAK DEMODULATION CIRCUIT

TABLE II. FAULT LIST

Finally, signals are induced, and decision table matrix $K$ is generated, as shown in Table III. In the example, $K^{\prime}$ is a 
$55 \times 14$ dimensional matrix. When induced, a $55 \times 12$ dimensional matrix $K$ is obtained. In order to easy to understand, a $8 \times 12$ dimensional sub-matrix is selected for analysis.

TABLE III. PART OF DECISION TABLE MATRIX

\begin{tabular}{|l|l|l|l|l|l|l|l|l|l|l|l|l|}
\hline & V1 & V2 & V3 & V4 & V5 & V6 & V1a & V2a & V3a & V4a & V5a & V6a \\
\hline no fault & 1 & 1 & 1 & 1 & 1 & 1 & 2 & 4 & 2 & 1 & 1 & 1 \\
\hline R1 short & 1 & 1 & 1 & 1 & 1 & 1 & 3 & 1 & 1 & 1 & 1 & 1 \\
\hline R1 open & 1 & 1 & 1 & 1 & 1 & 1 & 3 & 1 & 1 & 1 & 1 & 1 \\
\hline RV1 short & 1 & 1 & 1 & 1 & 1 & 1 & 1 & 3 & 1 & 1 & 1 & 1 \\
\hline RV1 open & 1 & 3 & 3 & 3 & 3 & 3 & 3 & 2 & 1 & 1 & 1 & 1 \\
\hline R2 short & 1 & 1 & 1 & 1 & 1 & 1 & 1 & 3 & 1 & 1 & 1 & 1 \\
\hline R2 open & 1 & 3 & 3 & 3 & 3 & 3 & 3 & 2 & 1 & 1 & 1 & 1 \\
\hline U1 Parameter Drifts & 1 & 2 & 2 & 2 & 2 & 2 & 2 & 5 & 3 & 1 & 1 \\
\hline
\end{tabular}

The relationship between fault and each test (conditional attributes) is described in decision table matrix. The testability modeling is finished when decision table matrix is generated.

By decision table matrix reduction, test points are optimized, and fault diagnosis strategy can be designed [13]. From Table III, we can see that decision table matrix is multivalued. Diagnosis strategy design algorithm based on extended multivalued dependency matrix can be used [14]. Moreover, the AND-THEN rules generated by rough set can be used in circuit fault detection and isolation. Aided by fault probability, testability analysis is achieved. In this example, 52 faults can be detected in decision table matrix.

\section{CONCLUSION}

In order to improve the repair and maintenance efficiency for circuit boards of certain equipment, a test and diagnosis system is designed. As the key technique of the system, a testability modeling and test generation method is put forward based on fault simulation and rough set. The testability information generated through fault simulation is processed with limitative and developmental discretization. Then, conditional attributes are reduced by rough set. And moreover, decision table matrix is provided. By decision table matrix reduction, test points are optimized, and fault diagnosis strategy can be designed. Moreover, the AND-THEN rules generated by rough set are used in circuit fault detection and isolation. Application proves that the system has high efficiency and precision.

\section{ACKNOWLEDGMENT}

This work is supported partly by the Twelfth Advanced Research Project under Grant No. 51319040102.

\section{REFERENCE}

[1] Sun Xiao-yun, Guo Li-wei and Sun Hui-qin. Design and Application of Virtual Instrument based on LabWindows/CVI [M]. Beijing: Electronic Industry Press, 2005.

[2] Min DUAN, Xi-en ZHANG, "A New method for Military Electronic Equipment Fault Knowledge Acquisition Based on Simulation", Computer Simulation, Vol 23, pp.10-14, April 2006 (In Chinese).

[3] Min DUAN, Xi-en ZHANG, "New method of Analog Circuit Fault Knowledge Acquisition Based on Simulation", Journal of System Sim ulation, Vol 18, pp.802-808, March 2006 (In Chinese).
[4] Min DUAN, Xi-en ZHANG, "Universal analog circuit fault knowledge acquisition flat base on simulation", Computer Engineering and Design, Vol 27, pp. 129-131,134, January 2006 (In Chinese).

[5] Min DUAN, Xi-en ZHANG, "New method of Analog Circuit Fault Knowledge Acquisition Based on Simulation", Journal of System Sim ulation, Vol 18, pp.802-808, March 2006 (In Chinese).

[6] Min DUAN, Xi-en ZHANG, "Universal analog circuit fault knowledge acquisition flat base on simulation", Computer Engineering and Design, Vol 27, pp. 129-131,134, January 2006 (In Chinese).

[7] C.E. Hymowitz L.G. Meares, B. Halal, New Techniques for Fault Diagnosis and Isolation of Switched Mode Power Supplies, Power Systems World, pp.21-36,1997.

[8] C.E. Hymowitz L.G. Meares, B. Halal, New Techniques for Failure Analysis and Test Program Design, IEEE AUTOTESTCON, pp.1231-1240, 1998.

[9] Zdislaw Pawlak, Rough Sets and Decision Algorithms,. RSCTC 2000, pp.30-45, 2000.

[10] Qing WANG, De-chun BA, Xiao-dong WANG, "Intelligent Fault Diagnosis Model Based on Rough Sets and Decision Tree Theory", Journal of Northeastern University(Natural Science), Vol 26, pp.284-287, January 2006 (In Chinese).

[11] Gen-zhu BAI, Zhi-li PEI, Jian WANG, Ying KONG, Li-sha LIU, "Attribute discretization method based on rough set theory and information entropy", APPLICATION RESEARCH OF COMPUTERS, Vol 25, pp.1701-1703, June 2008 (In Chinese).

[12] Xue-jun SU, Cheng-gang WANG, Shi-chuang CAI, "Testability Modeling and Analysis based on Rough Set", CSSS 2010, pp.2653-2656, June 2010.

[13] WANG Cheng-gang, ZHOU Xiao-dong, LIU Zhi-yuan, "Study on ATE Oriented Testability Analysis and Evaluation Method for Circuit Board", Vol 33, pp.521-524, August, 2010 (In Chinese).

[14] WANG Cheng-gang, SU Xue-jun, YANG Zhi-yong "Diagnosis Strategy Design based on Extended Multivalued Dependency Matrix", Journal of Engineering Design, Vol 17, pp.387-391, May, 2010 (In Chinese). 